\title{
“EFFICIENCY SPACE” A Framework for Evaluating Joint Evaporation and Runoff Behavior*
}

\author{
BY RANDAL Koster
}

\begin{abstract}
The relationship between evaporation and runoff at the land surface is captured in a simple "efficiency space" framework.
\end{abstract}

A land surface model (LSM) is an essential component of a full Earth system model. Past work shows that an LSM's evaporation and runoff formulations are inextricably linked (Koster and Milly 1997). Somewhat counterintuitively, an LSM with an excellent evaporation formulation will produce poor evaporation rates and correspondingly poor atmospheric states if its runoff formulation is inadequate. A reliable model treatment of land surface processes arguably requires an understanding of the evaporation-runoff connection.

In an online paper (briefly summarized here; available at http://dx.doi.org/I0.II75/BAMS -D-14-00056.2), a framework for investigating this connection is introduced. The framework captures

AFFILIATIONS: KOSTER-Global Modeling and Assimilation Office, NASA/GSFC, Greenbelt, Maryland CORRESPONDING AUTHOR: Randal Koster, Global Modeling and Assimilation Office, Code 6I0.I, NASA/GSFC, Greenbelt, MD 2077I

E-mail: randal.d.koster@nasa.gov

The abstract for this article can be found in this issue, following the table of contents.

DOI:10.1175/BAMS-D-14-00056.I

*This essay is a summary of a full article that is available online (10.1175/ BAMS-D-14-00056.2)

In final form 22 September 2014 the relationship between evaporation and runoff in a very simple and concise way: as a curve lying in "efficiency space."

The framework is built on the assumption that evaporation efficiency (the ratio of total latent heat flux $\lambda E$ to net radiation $R_{\text {net }}$, where $\lambda$ is the latent heat of vaporization) is a function of soil moisture $W$, averaged over the root zone,

$$
\lambda E / R_{\mathrm{net}}=\beta(W),
$$

and on the further assumption that runoff efficiency (the ratio of runoff production $Q$ over some time period to precipitation $P$ ) is also a function, to first order, of that same soil moisture:

$$
Q / P=F(W) .
$$

These are strong assumptions given the other factors controlling evapotranspiration and runoff generation (vegetation structure, topography, surface-subsurface soil moisture differences, etc.). In effect, the complexities not considered here are assumed to have homogeneous impacts and are thus implicitly captured in the forms of the functions $\beta$ and $F$. While such a framework is much too simple for general modeling applications, here the simplicity is considered to be a strength, as it allows a thorough understanding of the joint control of soil moisture over evaporation and runoff efficiency, the focus of the online paper. Simply put, the framework embodied by (1) and (2) captures succinctly the dual control of soil moisture 
on evaporation and runoff production. Perhaps surprisingly, given its simplicity, it indeed allows the accurate reproduction of observed annual streamflow variations, as shown in the online paper.

The two efficiencies (i.e., $\lambda E / R_{\text {net }}$ and $Q / P$ ) can be related to each other, as illustrated in Fig. 1a. The red curve on the right shows an arbitrary functional relationship between $\lambda E / R_{\text {net }}$ and $W$, and the blue curve directly below it shows an arbitrary relationship between $Q / P$ and $W$. For a soil moisture of 0.3 (degree of saturation), these functions produce an evaporation efficiency of about 0.3 and a runoff efficiency of about 0.05 . This pairing of evaporation and runoff efficiency values, along with every other possible pairing determined from the two functions, is plotted in efficiency space on the left side of Fig. 1a. The ef- ficiency space plot essentially shows how evaporation and runoff efficiencies vary with each other as the soil gets drier or wetter.

Naturally, a different set of functions would lead to a different curve in efficiency space. Figure $1 b$ shows another possibility. The number of possible efficiency function combinations is infinite; the corresponding $Q / P$ versus $\lambda E / R_{\text {net }}$ curves could lie anywhere in efficiency space, subject to the condition that $Q / P$ and $\lambda E / R_{\text {net }}$ increase monotonically with each other.

With this framework defined, the online paper aims to find the curve that best represents nature and thus best serves as a target for LSM development. Again, simple functional forms, such as those on the right in Fig. 1a, are, at best, crude approximations to the much more complex behavior seen in LSMs and in

a.
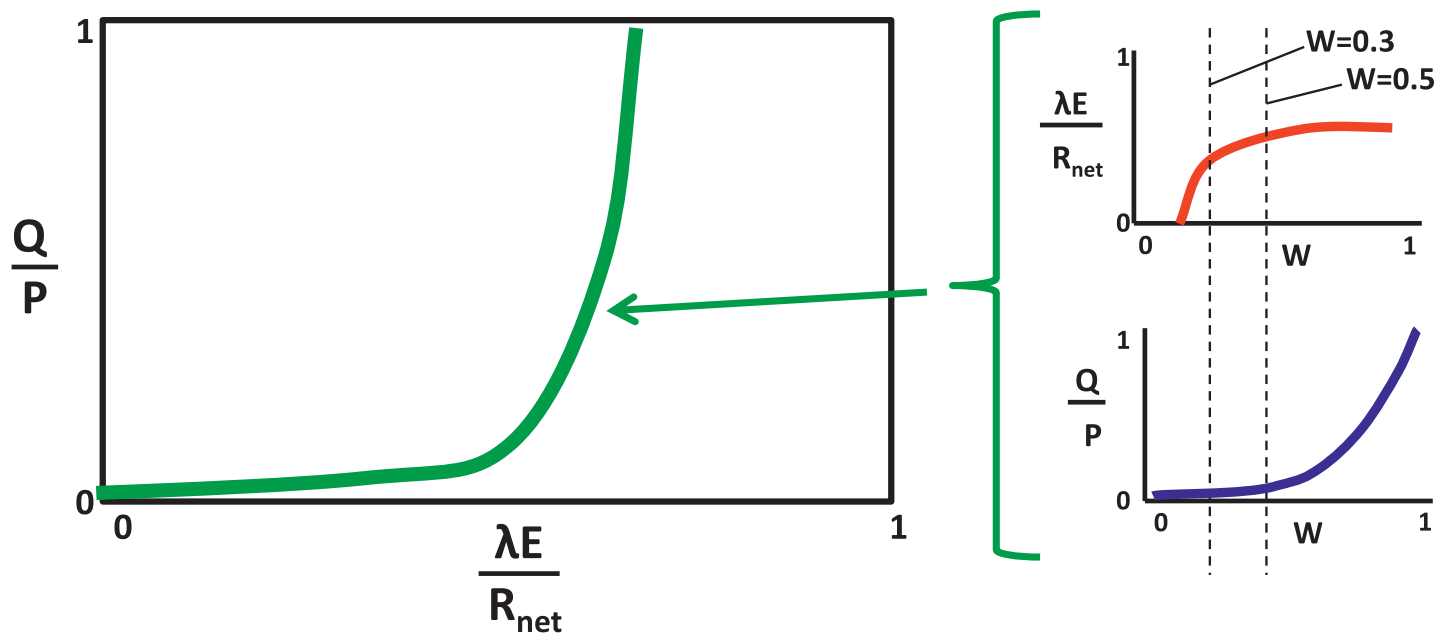

b.

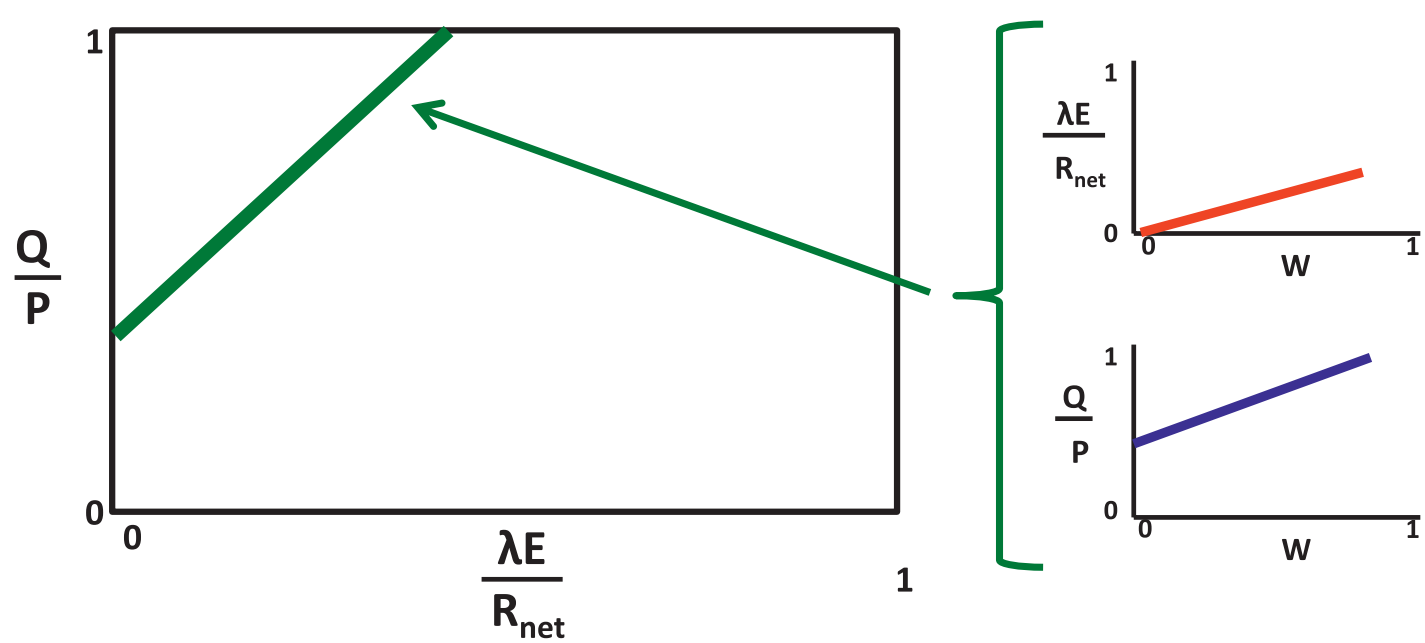

FIG. I. (a) Illustration of how a representative evaporation efficiency function ( $\lambda E / R_{\text {net }}$ vs $W$; the red curve on the right) and runoff efficiency function (QIP vs $W$; the blue curve on the right) combine to produce a single curve in efficiency space (the green curve on the left). (b) A second sample illustration. 
nature itself. Nevertheless, they capture enough of the underlying controls of soil moisture on evaporation and runoff to make the curve on the left meaningful and to allow nature's efficiency space curve, however it looks, to embody in a concise way an essential aspect of land surface hydrology.

\section{ANALYSIS OF EFFICIENCY SPACE WITH} A SIMPLE WATER BALANCE MODEL.

Following Koster and Mahanama (2012), a simple water balance model (WBM) is driven with observations-based forcing over the period 1948-2000 on a $2.5^{\circ} \times 2.5^{\circ}$ grid across the conterminous United States. The evaporation and runoff fluxes produced by the WBM at each daily time step are determined from user-defined evaporation and runoff efficiency functions (exemplified by those on the right-hand side of Fig. 1) acting on the WBM's soil moisture state variable. The final 52 years of simulated streamflow data are evaluated against observed streamflow data, which consist of naturalized stream gauge measurements in several large hydrological basins.

As described in the online article, a comprehensive analysis of WBM simulations shows that, in general, pairings of efficiency functions that produce the same curve in efficiency space generate essentially the same hydrological fluxes. For example, any number of pairings of evaporation and runoff efficiency functions could combine to produce the blue curve in Fig. 2a, but when each pairing is incorporated into the WBM, the resulting streamflows are very similar. This neatly reduces the dimensionality of the problem; efficiency space captures by itself the key controls of evaporation and runoff on simulated hydrology, without explicit consideration of how each flux varies with soil moisture.

The blue coloring of the curve in Fig. 2a is indexed to the low skill of the associated streamflow simulations; they are much too high relative to observed streamflows in the upper Mississippi basin. Pairings of evaporation and runoff efficiency functions that
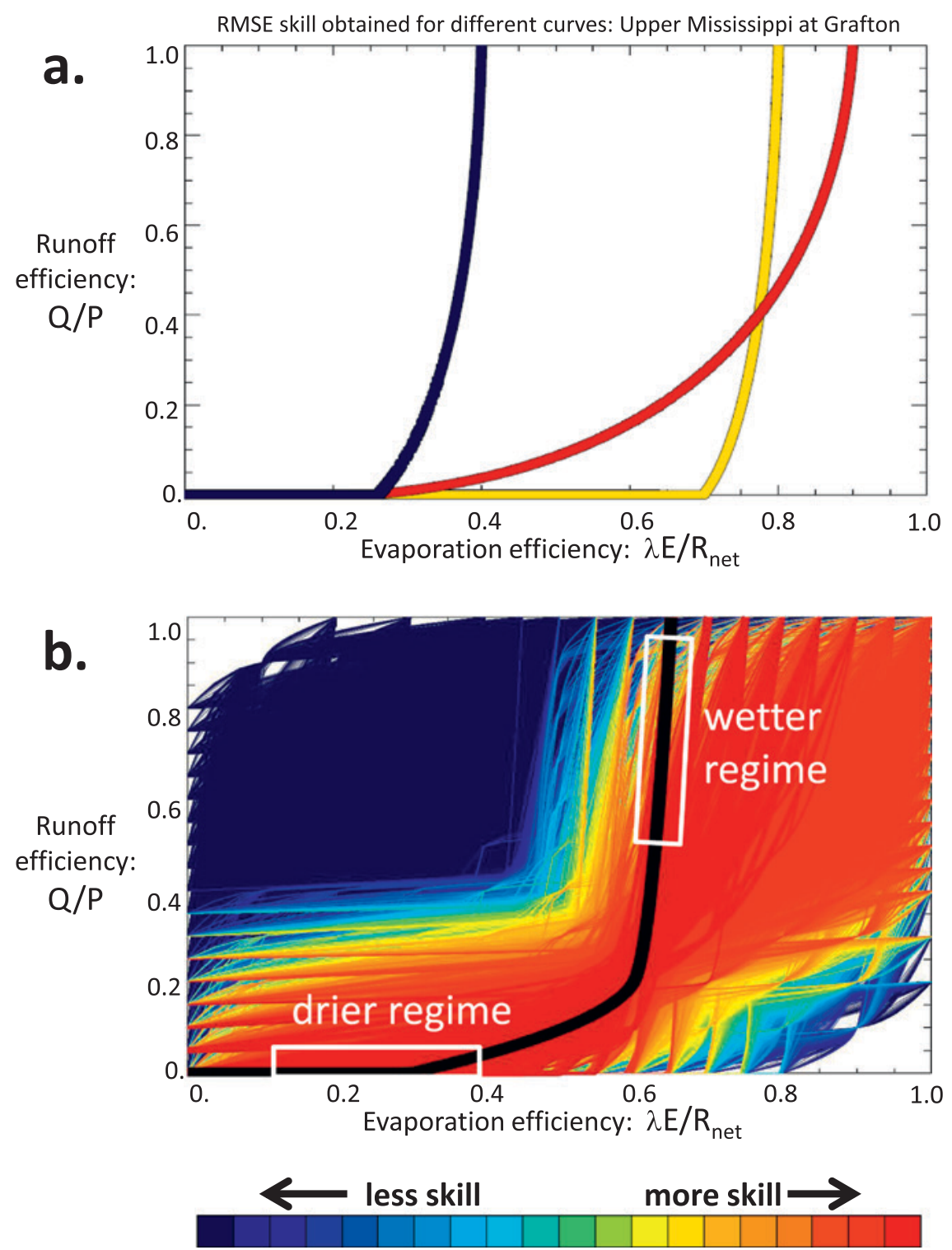

FIG. 2. (a) Three efficiency space curves, color-coded according to the degree to which corresponding WBM simulations reproduce observed streamflows in the upper Mississippi basin. The red curve corresponds to the most skillful simulation, and the blue curve corresponds to the least skillful simulation. Skill is quantified with an RMSE metric, though numbers are not shown in order to emphasize relative skill. (b) As in (a), but with over 23,000 color-coded curves overlain on the plot. The solid black curve is the curve associated with the greatest simulation skill. 
lead to the yellow curve in Fig. 2a produce streamflows that are somewhat more accurate, and pairings that map to the red curve produce highly accurate streamflows. Using this color-coding scheme for simulation accuracy and plotting higher-scoring curves on top of lower-scoring ones, Fig. $2 b$ shows the results of testing more than 23,000 curves in efficiency space. Figure $2 b$ (and similar plots for other basins) is a centerpiece result of the online paper. Given that observations are used to drive the WBM and to evaluate the skill levels, any dark red curve (corresponding to the highest skill values) is a reasonable representation of how evaporation and runoff efficiencies vary with each other in nature.

The heavy black line indicates the highest-scoring curve, though not by a large margin. Assuming that this particular curve reflects nature, we can infer the following for this basin: during dry conditions, runoff production does not begin significantly until the evaporation efficiency is about 0.3 and the sensitivity of runoff efficiency to changes in soil moisture is small relative to that of evaporation efficiency until the soil is wet enough to support an evaporation efficiency of about 0.6 , at which point the relative strengths of the sensitivities are reversed.

\section{RELEVANCE TO LAND MODEL} DEVELOPMENT. When evaluating the realism of an LSM, a key element to consider is the manner in which it allows variations of soil moisture to affect the magnitudes of evaporation and runoff. LSMs have built within them (implicitly) an effective relationship between evaporation and runoff efficiencies, represented by some curve in efficiency space. By examining an LSM's behavior through this diagnosed curve, the highly complicating issue of model dependence in the definition of the soil moisture state (Koster et al. 2009) is avoided.

Such LSM evaluations prove to be illuminating. A full LSM with an effective efficiency curve lying well above that determined for nature (e.g., above the black curve in Fig. 2b) is indeed found to overestimate streamflows relative to observations. Another LSM, this one with an efficiency curve lying below that for nature, is found to underestimate streamflows. The framework's chief advantage for such analyses is its ability to describe an LSM's deficiencies in streamflow production in terms of its joint evaporation and runoff behavior rather than solely in terms of runoff formulation. As described in the online article, the relative positions of the model and "observed" efficiency curves provide information on the direction needed for model development.

SUMMARY. A first-order understanding of the hydrology simulated by an LSM requires the joint analysis of its evaporation and runoff behavior. To explore this joint behavior and to provide a potentially useful tool for developing, evaluating, and improving LSM formulations, the concept of efficiency space is introduced. Analysis with a simple water balance model shows that for the upper Mississippi basin, nature's curve in efficiency space lies somewhere within the dark red region of Fig. 2b. Results for other basins are similar. In its own way, such a curve is conceptually powerful, as it captures, in a very succinct way, a critical relationship underlying the determination of annual hydrological fluxes.

The online article expands considerably on these concepts and describes in addition (i) the impact of soil reservoir depth on the efficiency space relationships, (ii) the use of the observed relationship to "tune" a full land surface model, and (iii) the connection between the efficiency space analysis and the landmark water and energy analysis of Budyko (1974). The online paper provides a more comprehensive argument for the potential use of the efficiency space framework in LSM development. In the long run, such development should lead to improvements in overall Earth system model performance.

\section{REFERENCES}

Budyko, M. I., 1974: Climate and Life. Academic Press, New York, 508 pp.

Koster, R. D., and P. C. D. Milly, 1997: The interplay between transpiration and runoff formulations in land surface schemes used with atmospheric models. J. Climate, 10, 1578-1591, doi:10.1175/1520 -0442(1997)010<1578:TIBTAR>2.0.CO;2.

- , and S. P. P. Mahanama, 2012: Land surface controls on hydroclimatic means and variability. J. Hydrometeor., 13, 1604-1620, doi:10.1175/JHMD-12-050.1.

—, Z. Guo, R. Yang, P. A. Dirmeyer, K. Mitchell, and M. J. Puma, 2009: On the nature of soil moisture in land surface models. J. Climate, 22, 4322-4335, doi:10.1175/2009JCLI2832.1. 